\title{
Cambios contemporáneos en el mundo del trabajo, condiciones de subjetivación y gubernamentalidad ${ }^{* *}$
}

\author{
Current Changes in the World of Work, Conditions \\ of Subjectification and Governmentality
}

\section{Mudanças contemporâneas no mundo do trabalho, condições de subjetivação e governabilidade}

\footnotetext{
* Candidata a Doctora en Psicología, Línea Psicología Social, Universidad del Valle. Becaria de Colciencias. Magíster en Sociología, Universidad del Valle. Psicóloga, Pontificia Universidad Javeriana-Cali. ORCID https://orcid.org/ooooooo2-9199-4383. Correo electrónico: deidi.maca@correounivalle.edu.co

** Psicólogo de la Universidad de Los Andes, Doctor en Psicología social por la Universidad Autónoma de Barcelona. Profesor Asociado de la Universidad del Valle, Director de la Línea de Investigación Narrativas, Subjetividad y Construcción Social. Secretario General de la Asociación Iberolatinoamericana de Psicología Política 2016-2018. ORCID https:// orcid.org/oooo-0002-0250-251X. Correo electrónico:nelson.molina@correounivalle.edu.co

${ }_{* * *}$ Este artículo de investigación hace parte de la tesis doctoral, en curso, titulada «Subjetividades emergentes a partir del emprendimiento como forma de gubernamentalidad neoliberal», que está siendo elaborada por la estudiante Deidi Maca, bajo la dirección del profesor Nelson Molina, y que tuvo el 3 de marzo de 2017 como fecha de inicio. Artículo de investigación recibido el 15-01-2018 y aceptado 06-04-2018.
} 


\section{Cómo citar}

Maca URbano, D. Y. y Valencia Molina, N. (2018).

Cambios contemporáneos en el mundo del trabajo, condiciones de subjetivación y gubernamentalidad. CS, (25), 137-166.

DOI: http://dx.doi.org/10.18046/recs.i25.2700 
El objetivo de este artículo es realizar una primera aproximación a las formas como parte de la literatura en Ciencias Sociales, aborda, a nivel conceptual y metodológico, algunas condiciones de subjetivación relacionadas con cambios contemporáneos en el mundo del trabajo desde una perspectiva de la analítica de la gubernamentalidad, e interesándose por los efectos en la subjetividad que esto conlleva. Para la búsqueda de los artículos se hizo uso del recopilador de bases de datos Scopus y como técnica de investigación se hizo uso del análisis de contenido. Se encontró una tendencia a considerar que las subjetividades son formadas/producidas por formas de gubernamentalidad, lo cual abre el camino a una discusión sobre subjetividades determinadas o subjetividades emergentes, es decir, a la consideración de cuáles formas de gubernamentalidad promueven u ofrecen condiciones de subjetivación a partir de las cuales emergerían ciertas subjetividades.

PALABRAS CLAVE:

Trabajo, gubernamentalidad, subjetividad

The objective of this article is to conduct a preliminary study of the ways in which some of the literature pertaining to the social sciences addresses, at a conceptual and methodological level, certain conditions of subjectification related to current changes in the world of work from the perspective of an analysis of governmentality focusing on in the effects on subjectivity that this implies. The research consisted of a content analysis of articles found on the abstract and citation data base Scopus. This analysis demonstrated that a trend exists that posits that subjectivities are produced by forms of governmentality. This in turn opens up a discussion of determinate subjectivities or emergent subjectivities, which is to say of taking into consideration which forms of governmentality foster or provide conditions of subjectification from which certain subjectivities emerge.

\section{KEYWORDS:}

Work, Governmentality, Subjectivity 
O objetivo deste artigo é realizar uma primeira aproximação às formas como parte da literatura - pertencente às ciências sociais - aborda, a nível conceitual e metodológico, algumas condições de subjetivação relacionadas com as mudanças contemporâneas no mundo do trabalho de uma perspectiva da analítica da governabilidade e interessando-se pelos efeitos na subjetividade que isso leva. Para a pesquisa dos artigos foi feito o uso de um recompilador de bases de dados Scopus e como técnica de pesquisa foi feito o uso de análise de conteúdo. Se encontra que há tendência a considerar que as subjetividades são formadas/produzidas por formas de governabilidade, o qual abre caminho em torno a uma discussão sobre subjetividades determinada ou subjetividades emergentes, como a consideração de formas de governabilidade promovem ou oferecem condições se subjetivação a partir das quais emergirão certas subjetividades.

\section{PALAVRAS CHAVE:}

Trabalho, governabilidade, subjetividade 


\section{Introducción}

Este artículo se realiza en el marco de una tesis doctoral en psicología en la que se propone que el emprendimiento es una forma de gubernamentalidad; es decir, que el emprendimiento, en tanto objeto de discurso polifónico y polivalente que puede remitir a autoempleo, a propiedad y dirección de negocio o a unidades de negocio legitimadas legal, institucional o socialmente, comprendería en principio políticas públicas, instituciones, actores y saberes que permitirían ejercer poder sobre la población a través de dispositivos de control; y traería una nueva especificación del sujeto de gobierno como agente activo de su propio destino que debe gobernarse a sí mismo de manera responsable, gestionar sus propios recursos, lograr autoasegurarse, autorregularse y buscar su autorrealización. En este contexto, emergerían formas particulares de pensar, sentir y actuar. Así que se propone estudiar un proceso de subjetivación particular a partir del emprendimiento como forma de gubernamentalidad.

El objetivo del presente artículo es realizar una primera aproximación a las formas como parte de la literatura en ciencias sociales, aborda, a nivel conceptual y metodológico, algunas condiciones de subjetivación relacionadas con cambios contemporáneos en el mundo del trabajo desde una perspectiva de la analítica de la gubernamentalidad, e interesándose por los efectos en la subjetividad que esto conlleva. Interesan los cambios contemporáneos en el mundo del trabajo, puesto que, en términos de contexto, el emprendimiento estaría instalado allí.

Para la búsqueda de los artículos se hizo uso del recopilador de bases de datos Scopus, en un periodo comprendido entre 2007 y julio de 2017, artículos escritos en inglés, español y portugués. Esta primera aproximación hace uso de Scopus puesto que permite acceder a diferentes bases de datos que recogen un volumen grande de las denominadas publicaciones de alto impacto; lo cual no quiere decir que sea el único medio de circulación de la literatura sobre el fenómeno de interés. La técnica de investigación se apoya en el análisis de contenido (Bardin, 2002). Los artículos que finalmente constituyen el universo de análisis se seleccionaron considerando que su abordaje empleara las nociones de gubernamentalidad y subjetividad para el estudio de diferentes fenómenos relacionados con el mundo del trabajo, que para los autores constituye las condiciones de subjetivación, y que tuvieran como sujetos a trabajadores o futuros trabajadores. Los artículos seleccionados pertenecen a los campos de ciencias sociales, psicología, administración y economía, definidos así por Scopus.

Las palabras clave de búsqueda fueron, en un primer momento: governmentality y subjectivity; y, en un segundo momento: work, governmentality y subjectivity. Al definir las palabras clave para la búsqueda nos dimos cuenta de que, usando tanto la primera ecuación de búsqueda como la segunda, aparecían artículos diferentes; 
así que se decidió emplear las dos. Usando la primera ecuación aparecieron un total de 349 artículos; y usando la segunda, un total de 94, lo cual es un primer reflejo de la escasa literatura sobre el tema de interés. Al emplear los primeros filtros, se llegó a 253 artículos con la primera ecuación; y a 71, con la segunda. Con un total de 324 artículos se inició el examen de los abstracts para configurar una primera base de artículos para revisión; se empezaron a excluir artículos que versaran sobre salud, arte, violencia, medio ambiente, consumo y mercado laboral. Después de la lectura de los abstracts se configuró otra base de artículos para su lectura completa. Durante la lectura fluctuante de estos artículos, se excluyeron los que tienen como foco las finanzas (Pellandini-Simányi, Hammer, y Vargha, 2015), el psy complex (Bailey, 2015; Matza, 2012), el espacio social (Dlaske, Barakos, Motobayashi, y McLaughlin, 2016; Zuckerwise, 2012) y las políticas públicas en educación (Dahlstedt y Hertzberg, 2012; Suspitsyna, 2010). De igual manera, se excluyeron las investigaciones en las que los sujetos de interés no son trabajadores (Gutiérrez Monclus y Pujol Tarrès, 2016; Hay, Kapitzke, y Cbse, 2009; Laalo y Heinonen, 2016; Lolich, 2011; Motobayashi, 2016; Varman, Saha, y Skålén, 2011; Wong, 2010).

A continuación se presentan, en primer lugar, los métodos empleados por los investigadores para el abordaje de sus investigaciones. Posteriormente, una descripción de algunos de los cambios contemporáneos en el mundo del trabajo, en los que encontramos diferentes condiciones de subjetivación. Seguido, se retoma lo que se encuentra en la literatura revisada con respecto a las nociones de gubernamentalidad y subjetividad. Y, finalmente, se plantean algunas ideas en torno a una discusión sobre subjetividades determinadas o emergentes.

\section{Sobre el método}

En las investigaciones revisadas se recurre a las personas y a los documentos como fuentes de información. Como personas se cuenta, por ejemplo, con funcionarios de un programa argentino de empleo dirigido a jóvenes: Programa Jóvenes con Más y Mejor TrabajoTrabajo-PJMYMT- (Assusa y Brandán Zehnder, 2014); profesores de escuela que asisten a un programa de formación en Brasil (Proformacão) (Bocchetti y Oliveira, 2012); jóvenes que realizan trabajo voluntario en un hospedaje en Finlandia (Dlaske, 2016); emprendedores sociales (Dey y Steyaert, 2016); trabajadores de una compañía telefónica en Suecia (Thunman, 2015); trabajadores que pertenecen al sector público en Nueva Zelanda (Nyamori, 2009); y diferentes miembros de la comunidad educativa -profesores, directivos y estudiantes-, tanto de escuelas como de universidades de Estados Unidos, Reino Unido y Suecia (Alves y Barreto, 2009; Ball, 2016; Ball y Olmedo, 2013; Morrissey, 2015; Niesche, 2010; Raaper, 2016; Thunman, 2015). 
Entre los documentos se cuenta con textos que hacen parte de la literatura prescriptiva (Eraranta y Moisander, 2011); documentos de los Centros de Trabajo en el Reino Unido y páginas web populares que incluyen publicidad y consejos en torno al trabajo (Boland, 2016); archivos del sector público local en Nueva Zelanda (Nyamori, 2009); correos entre el investigador y profesores de escuelas y universidades de Estados Unidos y el Reino Unido (Ball, 2016; Ball y Olmedo, 2013); documentos sobre políticas de evaluación del profesorado en instituciones de educación superior en el Reino Unido (Raaper, 2016) y en España (Amigot y Martínez, 2013); documentos propios de la política pública relacionados con las reformas en la industria que problematizan el futuro del trabajador industrial en Australia (Reich, 2008); y sobre el desarrollo de carrera en países de la Unión Europea (Bengtsson, 2011).

La recolección de información se realiza principalmente mediante aproximaciones documentales o aproximaciones etnográficas. Las primeras recurren al análisis de los documentos anteriormente mencionados (Amigot y Martínez, 2013; Ball, 2016; Ball y Olmedo, 2013; Boland, 2016; Eraranta y Moisander, 2011; Reich, 2008) y se interesan por conocer cómo el discurso ahí plasmado pretende formar/producir o promover/ofrecer diferentes subjetividades. Las segundas incluyen técnicas de recolección de información como la entrevista en profundidad, la observación participante, la conversación y los grupos de discusión (Dey y Steyaert, 2016; Dlaske, 2016; Faÿ, Introna, y Puyou, 2010; Hassoun, 2012; Nyamori, 2009; Thunman, 2015). Las aproximaciones etnográficas se interesan por abordar la cotidianidad de los trabajadores en sus espacios laborales, sus prácticas, las relaciones que entablan y por conocer su discurso en torno a la manera como las formas de gubernamentalidad, especialmente la gubernamentalidad neoliberal, forman/producen subjetividades; o en torno a cómo las formas de gubernamentalidad promueven/ofrecen diferentes subjetividades. Cabe mencionar que algunas de las investigaciones recurren tanto a aproximaciones documentales como etnográficas (Alves y Barreto, 2009; Bocchetti y Oliveira, 2012; Morrissey, 2015; Niesche, 2010; Nyamori, 2009; Raaper, 2016).

El análisis de la información se realiza mediante diferentes técnicas, por ejemplo, el análisis de discurso propuesto por Foucault (Bengtsson, 2011; Boland, 2016; Eraranta y Moisander, 2011; Raaper, 2016), la aproximación interpretativa crítica (Nyamori, 2009), el análisis temático (Thunman, 2015) y el análisis de discurso crítico propuesto por Fairclough, el cual tiende a ser un método dialéctico que hace posible explorar las relaciones entre el discurso y otros elementos de las prácticas sociales tales como los sujetos, los valores y los instrumentos (Raaper, 2016). En términos generales, todas las investigaciones revisadas recurren a los planteamientos clásicos de Foucault y de autores de los estudios de la gubernamentalidad para realizar sus análisis tanto de lo que tiene que ver con gubernamentalidad como lo que se relaciona con subjetividad. 
De la estrategia metodológica empleada por los investigadores se puede derivar la importancia de abordar elementos de política pública y aspectos del discurso y de la cotidianidad de los sujetos en el marco de diferentes formas de gubernamentalidad.

\section{Condiciones de subjetivación y cambios contemporáneos en el mundo del trabajo}

Los artículos revisados abordan el trabajo desde la perspectiva de la gubernamentalidad y se interesan por las implicaciones en la subjetividad que conllevan los modos de conducir la conducta. De este modo, aquí se consideran los fenómenos relacionados con el trabajo como condiciones de subjetivación. Si bien es cierto que, en términos generales, las investigaciones tienen como foco el trabajo, también se pueden encontrar ciertas especificidades con respecto a este. Es así como algunos estudios se centran en fenómenos relacionados con cambios contemporáneos en el mundo del trabajo, nuevas formas de trabajo, distintos espacios de trabajo y ciertos fenómenos que acaecen en dichos espacios.

Parte de los fenómenos relacionados con cambios contemporáneos en el mundo del trabajo es la externalización de tareas, específicamente vista como un componente de innovación abierta (Ettlinger, 2016). Otro de los fenómenos tiene que ver con las nuevas lógicas de la gestión estatal del trabajo/no trabajo orientada a la redefinición de la relación Estado/desocupados por fuera del marco de la generación de situaciones de salvataje (Assusa y Brandán, 2014). También aparecen las reformas recientes en la industria, las relaciones industriales y la formación en educación vocacional, junto con la problematización de las habilidades industriales del trabajador, lo cual trae consigo la formación del «trabajador como aprendiz» como una nueva subjetividad (Reich, 2008). Otro de los fenómenos que aparecen es la autogestión de la carrera como una forma de gobierno del self (Bengtsson, 2011) y, por último, el discurso de los consejos para buscar trabajo ofrecidos por ciertos programas y páginas web que forman sujetos como buscadores de trabajo: jobseekers (Boland, 2016).

Como nueva forma de trabajo aparece el trabajo voluntario en el campo del turismo: workaway es una expresión de esta tendencia. Esta nueva forma de trabajo, ubicada en el mundo contemporáneo y neoliberalizado, traería consigo la figura del workawayer como una nueva subjetividad cosmopolita que empieza a ser formada (Dlaske, 2016). Aparece también el emprendimiento social, frente al que algunos autores sugieren que da cuenta de una tecnología de poder a través de la cual los actores de la élite justifican la reducción del Estado en su rol de proveedor de bienestar público y el desplazamiento de la responsabilidad hacia los sujetos. Desde 
esta lógica, el emprendimiento social forma una ideología que promueve un ethos con una orientación hacia los negocios en diversas áreas como la salud y el cuidado social (Dey y Steyaert, 2016).

Como espacios de trabajo, aparecen la empresa flexible y la racionalidad neomanagerial de gobierno que la caracteriza, la cual resulta relevante para comprender las formas de subjetividad laboral que promueve y que permiten visibilizar, a su vez, las resonancias de la gubernamentalidad neoliberal y la figura del empresario de sí (Stecher, 2015). El sector público también aparece como un espacio de trabajo, específicamente sobre las racionalidades y mecanismos a través de los cuales los mercados han sido propuestos como alternativas o complementos a la provisión tradicional basada en el Estado de Bienestar (Nyamori, 2009). También aparecen las escuelas (Alves y Barreto, 2009; Niesche, 2010) o las universidades (Amigot y Martínez, 2013; Bill, 2017; Gómez, Jódar, y Bravo, 2015; Morrissey, 2015; Raaper, 2016); algunas investigaciones se centran tanto en escuelas como en universidades (Ball, 2016; Ball y Olmedo, 2013). Aparece también un programa de formación para profesores de escuela (Bocchetti y Oliveira, 2012). Cabe mencionar que las investigaciones centradas en estos espacios educativos están interesadas en abordar los modos de gubernamentalidad neoliberal que existen a su interior.

Como espacios de trabajo aparece también la organización (Eraranta y Moisander, 2011; Faÿ et al., 2010) y las dinámicas de integración trabajo (empleo)-vida que pueden gestarse (Eraranta y Moisander, 2011) o la forma como la tecnología de la información ha hecho posible que los sistemas contables de administración sean desplegados para gobernar los espacios más íntimos de la vida cotidiana en la organización (Faÿ et al., 2010). Otras investigaciones se centran en comparaciones entre la organización y la escuela (Hassoun, 2012), específicamente sobre el multitasking como forma de autotrabajo en el marco del neoliberalismo, y la forma como el estrés es percibido y manejado en sectores con mayor preponderancia de mujeres -escuela- y sectores con preponderancia de hombres -una compañía telefónica-, lugares de trabajo que han sido objeto de nuevos regímenes de gobernanza que enfatizan en los métodos de la administración de negocios y la orientación al cliente (Thunman, 2015).

\section{Gubernamentalidad}

En los artículos revisados se encuentran aportes de la analítica de la gubernamentalidad a la comprensión de las transformaciones contemporáneas del mundo del trabajo. En el marco de estos aportes, se resalta la definición de gubernamentalidad 
empleada por los investigadores, ciertas formas de abordaje y algunos aspectos referidos a la gubernamentalidad neoliberal, como su definición y la nueva concepción de sujeto que trae consigo.

En la literatura abordada parece haber consenso frente a la definición de gubernamentalidad, retomada de los planteamientos de Michel Foucault: se define como la forma en que la conducta de los individuos o grupos debe ser dirigida, lo cual puede hacerse a través de mecanismos disciplinarios o de control, y a través de la alineación de las elecciones individuales con racionalidades políticas específicas. El término conducta tiene una referencia específica a comportamientos y acciones, y tiene particular importancia para la noción de autoconducta (Ball y Olmedo, 2013; Eraranta y Moisander, 2011; Niesche, 2010; Nyamori, 2009). En algunas ocasiones se retoma también la definición de la noción a partir de los planteamientos de Mitchell Dean, así, la gubernamentalidad se define como una actividad más o menos calculada y racional emprendida por una multiplicidad de autoridades y agencias, empleando una variedad de técnicas y formas de conocimiento, que busca formar la conducta a través de trabajar nuestros deseos, aspiraciones, intereses y creencias, con un conjunto impredecible de consecuencias, efectos y resultados (Eraranta y Moisander, 2011).

Es así como, considerando las definiciones de gubernamentalidad, el gobierno no se refiere tanto a las estructuras políticas y administrativas del Estado moderno, sino más a la conducción de la conducta (Ball y Olmedo, 2013; Eraranta y Moisander, 2011). La noción foucaultiana de gubernamentalidad permite explicar las racionalidades de gobierno que interconectan las tecnologías de poder sobre los otros con las tecnologías o prácticas de sí, en regímenes cuyas racionalidades políticas están basadas en el liberalismo y el neoliberalismo (Alves y Barreto, 2009). El término gubernamentalidad señala, por tanto, la conexión entre técnicas de gobierno de los otros y las de gobierno de sí mismo dentro de un dispositivo histórico. Nos permite pensar los efectos propios de determinada tecnología de poder, no solo en relación con la conducción de la conducta de los otros, sino vinculándola con las prácticas que el individuo es llevado a realizar sobre sí; es decir, en términos sociales, intersubjetivos y subjetivos (Amigot y Martínez, 2013). De esta manera, las intervenciones gubernamentales no administran individuos preexistentes, sino que forman sujetos a través de la inculcación de ciertas formas de conducta (Boland, 2016).

Es así como los estudios de la gubernamentalidad se enfocan en la intervención del Estado a través de organizaciones, procedimientos, reglas y categorías, incluyendo también formas no estatales de control institucional como, por ejemplo, las fábricas. Otros focos son las políticas públicas, los discursos expertos y el despliegue instrumental del conocimiento (Boland, 2016). Con respecto a las políticas públicas, 
la lógica político-institucional, en tanto racionalidad propia del Estado, posee como herramientas privilegiadas de gestión de lo social: la ley y el aparato administrativo. A través de ellas, opera y se realiza la gestión del capital, de poblaciones, de mercancías, de cosas, de espacios y de lugares. En este sentido, las políticas públicas son dispositivos centrales para esta lógica, junto a la ley y el aparato represivo, policial y legal, que asegura su cumplimiento; y por lo tanto se constituyen en mecanismos de poder (Assusa y Brandán, 2014).

Pasando a las formas de abordaje, Stecher (2015) plantea que orientar una investigación empírica desde la perspectiva de la analítica de la gubernamentalidad exige abordar tres ejes: genealógico, arqueológico y de la relación del sujeto consigo mismo. Como parte del abordaje desde la perspectiva de los estudios de la gubernamentalidad, otros autores distinguen tres dimensiones: racionalidades, tecnologías y efectos subjetivos. Las racionalidades pueden ser entendidas como una clase particular de lógicas de gobierno (Dlaske, 2016). Estas dan cuenta de formas calculadas mediante las cuales las mentalidades se inscriben en un régimen de prácticas, en este caso, nuevas prácticas de trabajo (Ettlinger, 2016).

Sobre las tecnologías, Gómez et al. (2015) señalan que la forma hegemónica de relacionarse con el conocimiento que intentamos problematizar, donde este se aleja de su dimensión social, responde a un conjunto de discursos y prácticas que, utilizando herramientas foucaultianas, denominaremos tecnologías y que modifican la subjetividad en relación con determinados objetivos políticos. Desde la perspectiva de la gubernamentalidad, a través de dispositivos compuestos de elementos heterogéneos (vocabularios, objetos, prácticas, sistemas de juicio, espacios) que construyen nuestra interioridad, se persigue un objetivo más o menos consciente de regulación social. La potencia del dispositivo o tecnología reside precisamente en el ensamblaje o agenciamiento de sus elementos que, de forma conjunta, son capaces de producir efectos de gobierno de la subjetividad (Gómez et al., 2015).

De esta manera, las tecnologías pueden ser pensadas como medios, mecanismos e instrumentos diversos y heterogéneos, los cuales posibilitan una orquestación calculada de actividades humanas bajo una racionalidad práctica dirigida hacia ciertas metas (Dlaske, 2016). Cabe mencionar que las tecnologías del self se intersectan con las tecnologías de dominación (Nyamori, 2009). Las primeras permiten a los individuos, por sus propios medios, llevar a cabo cierto número de operaciones en sus cuerpos, mentes y estilos de vida, tanto como transformarse a ellos mismos con el fin de lograr ciertos estados de felicidad y calidad de vida (Thunman, 2015). 


\section{Gubernamentalidad neoliberal}

En los artículos revisados se encuentran diferentes términos para hacer referencia a la gubernamentalidad neoliberal, como el neoliberalismo y el liberalismo avanzado. Las definiciones son retomadas de los planteamientos tanto de Michel Foucault como de Nikolas Rose, Mitchell Dean, John Girdwood, Thomas Lemke y Graham Burchell. Así, desde la analítica de la perspectiva de la gubernamentalidad, el neoliberalismo se define como una forma de vida en la que el mercado impregna todos los aspectos de la fábrica social (Ettlinger, 2016). El neoliberalismo constituye una mentalidad de gobierno, una concepción de cómo las autoridades deberían usar sus poderes con el fin de mejorar el bienestar nacional, los fines que deberían buscar, los males que deberían evitar, los medios que deberían usar y, crucialmente, la naturaleza de las personas sobre las cuales deberían actuar. El neoliberalismo se refiere entonces a estilos específicos de la mentalidad general de gobierno (Reich, 2008).

De acuerdo con Ball y Olmedo (2013), el neoliberalismo da cuenta de un nuevo régimen de verdad. Aunque comparte ciertos principios con el liberalismo clásico, el neoliberalismo difiere sustituyendo la lógica del intercambio por la lógica de la competencia. Esto representa un cambio epistemológico más amplio que implica una nueva antropología: el homo economicus, caracterizado por ser un empresario de sí mismo (Ball y Olmedo, 2013). Así, el neoliberalismo es un modo de gobierno que está arraigado en el discurso económico de la competencia; es un modo de gobierno que, por un lado, cree en la libre elección y en la primacía del mercado y, por otro, impone un incremento del escrutinio para facilitar el éxito institucional en las relaciones de competencia (Raaper, 2016).

Ball y Olmedo (2013) señalan que el neoliberalismo está allá y acá, afuera y adentro; se hace realidad y es constituido dentro de prácticas de la vida cotidiana, y nos hace hablar y actuar a través de nuestro lenguaje, propósitos, decisiones y relaciones sociales. Reich (2008) plantea que las características clave de las formas liberales avanzadas de gobierno incluyen la marketización de la vida económica, la fragmentación de lo social en una multiplicidad de mercados, la pedagogía disciplinaria para el perpetuo entrenamiento, el desplazamiento de empleados a emprendedores de sí mismos y la preponderancia de la forma-empresa. Otra característica del ensamblaje de los regímenes de gobierno liberal avanzado es el gobierno a distancia. Estos regímenes desplazan el discurso autoritario a otras prácticas sociales e institucionales a través de la seducción y la movilización de agentes y agencias dentro y más allá del Estado utilizando formas neoburocráticas del gobierno a distancia.

En el liberalismo avanzado gobernar es un arte que envuelve formas de poder que son transmitidas a través de la producción de subjetividades. A diferencia de 
regímenes previos de gobierno que estaban basados en formas de poder más autoritarias y jerárquicas, el gobierno en el liberalismo avanzado envuelve un ejercicio de poder que depende de la introducción de un ethos de responsabilidad a través de varias medidas de empoderamiento individual. En la gubernamentalidad neoliberal las formas políticas son extendidas a formas de automejoramiento y, así, la gubernamentalidad es menos un asunto de dominación de las personas que de optimización de su capacidad para la producción social haciendo de estas personas aptas, flexibles y autónomas (Dey y Steyaert, 2016).

Con respecto a la nueva concepción de sujeto que trae consigo el neoliberalismo, el sujeto neoliberal surge durante una significativa recalibración del pensamiento gubernamental durante el siglo XX. En las clases de 1978-1979, Foucault plantea que el ethos del gobierno estaba cambiando con el fin de producir sujetos como actores económicos autosuficientes, responsables de sus propios destinos, y cada acción humana se convertiría así en un proceso de inversión en sí mismo (Hassoun, 2012). En el marco de la gubernamentalidad neoliberal, se espera que los individuos lleven a cabo el self-management tomando responsabilidad personal y ejerciendo su capacidad de reflexión y cálculo, esto es, ejerciendo la autorregulación; sin embargo, esta es una libertad regulada, cuyo objetivo no es el logro de la autonomía, sino inducir a los individuos a movilizar sus recursos y capacidades e involucrarlos en una evaluación continua, mejoramiento y control de sus acciones (Thunman, 2015).

De esta manera, el ejercicio del poder apela directamente a la responsabilidad e implicación voluntaria de los individuos: el sujeto de gobierno es un sujeto libre y autónomo. Ya no se trata de producir sujetos dóciles y disciplinados a través de la coacción, sino de producir una subjetividad completamente implicada en la actividad que debe llevar a cabo. Al igual que en otros ámbitos, la lógica neoliberal no pretende anular la iniciativa de los gobernados sino trabajar sobre ella continuamente para utilizarla a su favor; la libertad se convierte en exigencia y mandato, se produce, organiza y administra (Gómez et al., 2015). Los aparatos del neoliberalismo son seductores, fascinantes y predominantemente necesarios. Es un nuevo sistema moral que subvierte y nos reorienta a sus verdades y fines, nos hace responsables por nuestro desempeño y por el desempeño de los otros; estamos cargados con la responsabilidad de actuar, y si no lo hacemos estamos en peligro de ser vistos como irresponsables. Así, estamos producidos más que oprimidos, estamos animados más que constreñidos (Ball y Olmedo, 2013).

De este modo, los nuevos discursos que valoran el riesgo inherente a la existencia individual y colectiva apelan a la capacidad de los sujetos para hacer elecciones; para alcanzar resultados se apoyan en la convicción (y la recrean) de que, al igual 
que sucede en otros espacios, la protección de derechos o regulaciones externas y colectivas son dañinos para la creatividad, la innovación y la empleabilidad, y son interpretados como corporativismo o falta de compromiso. La crítica a la burocracia disfuncional actúa como justificación de la desregulación. En esta batalla simbólica, frente a las inercias burocráticas se defiende una ética emprendedora, así como la necesidad de flexibilidad y continuo crecimiento (Gómez et al., 2015).

En algunos artículos se retoman ciertos elementos de la teoría del capital humano propuesta desde la concepción neoliberal norteamericana y abordados por Foucault (2007), teoría que remite a la idea del homo economicus como empresario de sí mismo, elemento clave de la nueva especificación del sujeto de gobierno. La consideración de los individuos como capital implica una particularidad en esta noción, no es un capital como los demás: la aptitud de trabajar, la idoneidad, el poder hacer algo como elementos definitorios de ese capital implican que es el propio trabajador el que aparece como si fuera una empresa para sí mismo. Por tanto, es ese poder hacer y esa dimensión agente del individuo la que tiene que ser modelizada y funcionar de maneras específicas. No es reprimida ni suprimida, es esperada, espoleada y alentada.

De la misma forma que la racionalidad liberal necesita libertad y debe producirla, las lógicas del nuevo capitalismo necesitan capacidad de actuar y de actuarse de manera motivada, y la promoción y mantenimiento subjetivo de mecanismos afectivos de (auto) control (Amigot y Martínez, 2013). Se puede pensar que la gubernamentalidad contemporánea amplía el espacio instrumentalizado e incitado de la subjetividad a la dimensión afectiva del capital humano. Si en la sociedad industrial estaba en juego, en primer lugar, la extracción de fuerzas físicas de los obreros, en la actualidad se ha ampliado hacia la dimensión cognitiva, aptitudinal y emocional (Amigot y Martínez, 2013; Ettlinger, 2016).

La noción de empresario de sí, de un modo equivalente a lo ya planteado del homo economicus, busca dar cuenta de las particulares modalidades de asujetamiento y subjetivación que caracterizan a los procesos de subjetificación bajo las coordenadas históricas de la gubernamentalidad neoliberal. Se trata de un perfil de sujeto fuertemente individualizado, emprendedor, responsable de su destino, que vive expuesto al riesgo y que debe ser capaz de reinventarse permanentemente (Stecher, 2015).

Como se planteó anteriormente, parece existir un consenso con respecto a los autores que se emplean como referentes. Es así como la aproximación a la gubernamentalidad es inspirada en los textos clásicos de Michael Foucault y en los estudios neofoucaultianos de gubernamentalidad, entre los que se destacan obras como The Foucault Effect: studies in governmentality (Burchell et al., 1991), Governmentality Studies in Education (Peters et al., 2009) y Governmentality: current issues and future challenges (Bröckling et al., 2011), los cuales problematizan diferentes formas de 
gubernamentalidad neoliberal y aspectos de la biopolítica del gobierno. De igual manera, habría consenso en la definición de las nociones de gubernamentalidad y de gubernamentalidad neoliberal.

Así la gubernamentalidad da cuenta del modo de dirigir la conducta de individuos o grupos, y la gubernamentalidad neoliberal hace alusión a la forma en que se establecen las relaciones entre la economía, el Estado y la sociedad en las que la primera juega un papel fundamental. El neoliberalismo crea entonces una nueva relación entre el Estado, la sociedad y la economía, y trae consigo una nueva concepción de sujeto. Como aspectos característicos de la relación entre Estado y economía se destaca el gobierno mediante la acción a distancia; y como elementos centrales de la relación entre sociedad y economía, sobresalen la reestructuración del gobierno social en nombre de una lógica económica y la creación y el sostenimiento de los elementos centrales del bienestar económico -la forma empresa y la competenciapor parte del gobierno económico; además de la desgubernamentalización del Estado y la desestatalización del gobierno.

No obstante, en la literatura revisada parece haber un mayor interés en lo que respecta a la nueva concepción de sujeto de gobierno que trae consigo el neoliberalismo que en las transformaciones de la relación economía, Estado y sociedad. El neoliberalismo no solo trae consigo una nueva relación entre el Estado, la sociedad y la economía, sino que, derivado de ello, también trae consigo una nueva especificación del sujeto de gobierno como agente activo que debe gobernarse a sí mismo de manera responsable, gestionar sus propios riesgos, lograr autoasegurarse, autorregularse (Castro-Gómez, 2015; Foucault, 2007) y buscar la autorrealización (Rose, 1996). Este énfasis quizá esté relacionado con el interés de los investigadores, el cual reside en los efectos a nivel de la subjetividad que el neoliberalismo conlleva.

\section{Algunos casos}

Quizá donde se encuentra un mayor despliegue de la analítica de la gubernamentalidad es en los casos que son abordados por los investigadores. Como se menciona párrafos arriba, la analítica de la gubernamentalidad es empleada para abordar diferentes aspectos relacionados con el trabajo. Así, algunos estudios se centran en fenómenos relacionados con cambios contemporáneos en el mundo del trabajo, nuevas formas de trabajo, distintos espacios de trabajo y ciertos fenómenos que acaecen en dichos espacios.

Uno de los fenómenos relacionados con los cambios contemporáneos en el mundo del trabajo tiene que ver con las nuevas lógicas de la gestión estatal del trabajo/ no trabajo. En este caso, los investigadores abordan la formulación del Programa 
Jóvenes con Más y Mejor Trabajo-PJMYMT-, como una política activa que intenta producir una forma singular de subjetividad a partir de su intervención en el mundo del trabajo con criterios de ciudadanía/inclusión y generar una nueva trama institucional que se condice con el rol de promotor y orientador asumido por el Estado. El nuevo paradigma de políticas activas posiciona al Estado en un lugar diferente en relación a los problemas del empleo, que lo desplazan desde un modelo de Estado asistencialista hacia un modelo de Estado promotor (Assusa y Brandán, 2014).

Otro de los fenómenos que aparecen es la autogestión de la carrera como una forma de gobierno del self. En este caso se encuentran ciertas racionalidades políticas y tecnologías de gobierno que buscan cambiar la mentalidad del desarrollo de carrera y la reformación de la orientación de carrera como un instrumento social de gobierno de la población. De esta forma, la política de orientación de carrera busca formar no solo una fuerza de trabajo competitiva, sino también ciudadanos responsables y emprendedores (Bengtsson, 2011). Por último, los consejos para los jobseekers pueden ser considerados discursos que ejercen un poderoso efecto en el gobierno de su conducta y su formación como sujetos, particularmente a través de la prescripción de ciertos ejercicios de autoevaluación, control y presentación que equivalen a técnicas del self, como las búsquedas, las aplicaciones, las presentaciones, las entrevistas, la elaboración de hojas de vida y las redes de contacto (Boland, 2016).

Como espacios de trabajo aparecen la empresa flexible y la racionalidad neomanagerial de gobierno que la caracteriza, la cual resulta relevante para comprender las formas de subjetividad laboral que promueve y que permite visibilizar, a su vez, las resonancias de la gubernamentalidad neoliberal y la figura del empresario de sí (Stecher, 2015).

Como fenómenos que ocurren en la organización como espacio de trabajo, se resalta la forma como las tecnologías de la información han hecho posible que los sistemas contables de administración-MAS- sean desplegados para gobernar los espacios más íntimos de la vida cotidiana en la organización. De esta manera, como un régimen de gubernamentalidad, MAS constituye un modo de acción que no actúa directa ni inmediatamente sobre los otros, sino sobre sus acciones, presuponiendo la libertad de actuar en una forma u otra (Faÿ et al., 2010). En estos espacios de trabajo se resalta también la aparición del multitasking, el cual parece menos un régimen de autogobierno que una borrosa selección de técnicas, directivas y advertencias a través de las cuales los sujetos se hacen cargo del estrés de monitorear su propio uso tecnológico (Hassoun, 2012). De igual modo, aparecen las prácticas administrativas para el manejo del estrés que median las formas en las que los empleados pueden llegar a gobernar su propia subjetividad a través de las prácticas de administración del estrés, prácticas que están implicadas en el ejercicio diario del poder sobre el self(Thunman, 2015). 
El sector público también aparece como un espacio de trabajo, específicamente lo que tiene que ver con las racionalidades y mecanismos a través de los cuales los mercados han sido propuestos como alternativas o complementos a la provisión tradicional basada en el Estado de Bienestar, cambios asociados con los esfuerzos por constituir trabajadores de las autoridades locales como sujetos business-like a través de mecanismos disciplinarios y tecnologías del self (Nyamori, 2009).

También aparecen las escuelas o las universidades como espacios de trabajo. Las investigaciones que se centran en estos espacios educativos están interesadas en abordar los modos de gubernamentalidad neoliberal que existen a su interior. Con respecto a las escuelas, se encuentra que el neoliberalismo instituye racionalidades de gobierno que estimulan nuevas formas de gerenciamiento o autogerenciamiento que suponen la participación activa de los profesores en su propia sujeción (Alves y Barreto, 2009). La perspectiva de la gubernamentalidad puede ser útil para exponer las características de algunas prácticas educativas que pueden resultar más en tecnologías efectivas de control que en la naturaleza democrática del self-management (Niesche, 2010). La gubernamentalidad estaría atenta, igualmente, en la producción educativa (Bocchetti y Oliveira, 2012).

Con relación a las universidades, encontramos que algunas investigaciones consideran de manera crítica las prácticas de gubernamentalidad neoliberal en la academia contemporánea (Morrissey, 2015). Otras se centran en las prácticas evaluadoras (Gómez et al., 2015) o en las coordenadas que enmarcan hoy la tarea investigadora y que responden a la incorporación de políticas neoliberales en la universidad. Dichas políticas constituyen formas de gubernamentalidad que han modificado tanto el valor y el uso que se le da al conocimiento como la subjetividad de los colectivos universitarios (Gómez et al., 2015).

\section{Subjetividad}

Con respecto a la noción de subjetividad, se puede decir que sus definiciones son escasas, y en ocasiones se usan los términos sujeto, selfy subjetificación como términos relacionados. Se encuentra que la subjetividad es abordada, principalmente, desde una perspectiva foucaultiana; y que los investigadores consideran fundamental la tensión entre poder y resistencia para comprender la formación de subjetividades.

En este sentido, la definición propuesta por Ball y Olmedo (2013) retoma las ideas de Michel Foucault y hace alusión a la subjetividad como un proceso de transformación que se enfoca en lo que nosotros somos, es decir, en el trabajo del cuidado de sí. En este sentido, no hay un sujeto que ya esté formado, y el self no es 
solo una constante de inicio, sino también una constante de fin, en el doble sentido de la palabra, como entidad consumada y como objetivo concebido (Ball y Olmedo, 2013). De esta manera, esta definición de subjetividad lleva a la noción de self. Estos autores recurren también a la noción de sujeto. Para Foucault, la palabra sujeto tiene dos sentidos, ambos sugieren una forma de poder que subyuga y hace al sujeto. Por un lado, el término sujeto está relacionado con el estado de sujeción a alguien, ya sea por control o por dependencia. Por otro lado, el término sujeto se refiere a la autoconfiguración de una identidad por conciencia y autoconocimiento. Es así como el sujeto no es una simple sustancia, sino una forma (Ball y Olmedo, 2013).

Por su parte, Bengtsson (2011) sugiere que la subjetividad está relacionada con el proceso de autoformación y autoconocimiento, y es formada a través de las tecnologías del self y las racionalidades políticas. Los individuos son interpelados como sujetos de cierta manera por un complejo de ideas, prácticas y técnicas en relaciones específicas de poder y conocimiento, y se vuelven sujetos gobernables a través de posiciones subjetivas disponibles en ciertos contextos históricos (Bengtsson, 2011). Cada subjetividad del individuo en cualquier tiempo depende de la interacción entre su historia personal a través del tiempo, el espacio y el contexto (Ettlinger, 2016).

Raaper (2016) emplea la noción de subjetificación y la define como el proceso de volverse un sujeto dentro de una producción discursiva de poder/conocimiento; tiende a un proceso sin fin en el cual las posiciones subjetivas son creadas, negociadas, aceptadas y transformadas en y a través de prácticas discursivas cotidianas. Para Foucault, las relaciones de poder forman las subjetividades que existen en relaciones complejas en la red total de lo social; plantea que la vida social es construida en una forma en la cual alguien puede actuar sobre otros; sin embargo, también enfatiza en que el poder puede ser actuado únicamente en sujetos libres, sujetos que tienen una variedad de formas de responder y comportarse. Esto significa que poder y resistencia/libertad existen en una relación contradictoria pero fundamental (Raaper, 2016). Esta relación entre poder y resistencia va a ser clave para la comprensión de la formación de subjetividades, como se verá más adelante.

Como se menciona párrafos arriba, hay una tendencia marcada a emplear la perspectiva foucaultiana para abordar la subjetividad. Solo en un caso los autores emplean la perspectiva fenomenológica. Con respecto a la primera, Stecher (2015) plantea que es desde la trípode analítica -autoridad/poder, juegos de verdad y subjetivación- que la analítica de la gubernamentalidad incorpora los tres grandes momentos o perspectivas de análisis de la obra de Foucault -arqueología, genealogía, ética-, ofreciendo un marco conceptual fecundo para analizar los procesos de producción de subjetividades en diferentes campos de relaciones sociales al interior de las sociedades modernas. Se trata de una perspectiva anitiesencialista, 
antidualista y crítica que permite visibilizar el modo en que las formas de subjetividad presentes en un particular campo social y escenario sociohistórico están vinculadas a las racionalidades de gobierno imperantes (Stecher, 2015). Con respecto a la segunda, los autores muestran que la fenomenología, especialmente el trabajo de Michel Henry, contribuye a entender cómo los actores viven sus vidas a través de la simultaneidad de sistemas de abstracción y su efecto encarnado y situado en la praxis diaria (Faÿ et al., 2010).

\section{Poder y resistencia}

La gubernamentalidad opera a través del ensamblaje y propuesta de formas particulares de subjetividad frente a las cuales pueden darse tanto procesos de adscripción como procesos de resistencia. Se encuentran diferentes formas de nombrar esta relación: neurosis y resiliencia (Ettlinger, 2016); ajuste y resistencia (Eraranta y Moisander, 2011); poder y resistencia (Ball y Olmedo, 2013); y determinación y agencia (Dey y Steyaert, 2016; Morrissey, 2015). Con respecto a esta última relación, Foucault reintroduciría un sentido de la agencia individual en su trabajo sobre el poder, enfatizando las prácticas de sí mismo a través de las cuales los individuos se constituyen ellos mismos como sujetos en su propio conocimiento (Dey y Steyaert, 2016). Plantear que el sujeto es constituido no implica plantear que es determinado, por el contrario, el carácter constituido del sujeto es la precondición de su agencia. En la medida en que el proceso de constitución de los sujetos está lleno de conflictos y contradicciones, hay espacios para la resistencia (Morrissey, 2015).

En su trabajo temprano sobre el poder, Foucault se preguntaba por la manera en que somos constituidos como sujetos rendidos a relaciones de poder. Su trabajo ético complementa este foco planteando la pregunta si y cómo los individuos son capaces de desestabilizar el llamado del poder transgrediendo creativamente la subjetividad que les es ofrecida (Dey y Steyaert, 2016). En las relaciones de poder hay necesariamente posibilidad de resistencia (de resistencia violenta, huida, decepción, estrategias capaces de revertir la situación), porque si no hubiera posibilidad de resistencia no podría haber relaciones de poder (Ball y Olmedo, 2013). El poder no siempre es represivo, hay también espacios para la acción y para la resistencia (Niesche, 2010). La reconfiguración del sujeto de gobierno confiere obligaciones y deberes, al mismo tiempo que abre nuevos espacios de decisión y acción (Ball y Olmedo, 2013).

Cabe mencionar que la contra-conducta constituye una forma de resistencia, la cual da cuenta de la lucha contra los procesos implementados para conducir a otros (Bill, 2017). Otra forma de hacer alusión a los procesos de resistencia es a partir de la noción de lucha. Esta implica un compromiso con-y puede implicar un rechazo de- 
la gubernamentalidad neoliberal en sus propios términos. Es decir, todos aquellos en quienes se ejerce el poder, todos aquellos que lo encuentran intolerable, pueden comenzar la lucha en su propio terreno y sobre la base de su propia actividad (o pasividad). El punto es que en las economías neoliberales, los sitios de gobierno y los puntos de contacto también son sitios para la posibilidad de rechazo. Sin embargo, el punto de partida para unas políticas de rechazo es el sitio de la subjetividad. Es una lucha en contra de lo que nos hemos convertido, de lo que no queremos ser (Ball, 2016).

De lo anterior se deriva entonces la importancia de considerar la subjetividad a partir de estas relaciones y tensiones entre poder y resistencia y las diferentes subjetividades que se pueden encontrar en medio, tal como lo plantea Ettlinger (2016).

\section{Subjetividades contemporáneas: algunos casos}

Como se señala anteriormente, en la literatura revisada se encuentran ciertos vacíos en la conceptualización de la noción de subjetividad, lo cual exige, para futuras investigaciones, un trabajo de conceptualización fuerte que retome tanto elementos de la perspectiva foucaultiana, como de otras, como el construccionismo social. Quizá donde se encuentra un mayor despliegue es en los casos estudiados por los investigadores. A continuación, se presentan ciertas implicaciones subjetivas y algunas formas y posibilidades de resistencia.

Se puede señalar que las formas de gubernamentalidad en las que los sujetos están inmersos en los distintos fenómenos, espacios, programas y formas de trabajo conllevan algunas implicaciones a nivel de su subjetividad y es por esto que hablamos de condiciones de subjetivación presentes en fenómenos relacionados con el mundo del trabajo.

En el campo de las organizaciones, Stecher (2015) destaca cuatro aspectos de la racionalidad de gobierno de la empresa flexible que son relevantes para comprender las formas de subjetividad laboral que promueve: el tránsito del asalariado al empresario de sí, que invierte su capital humano; la integración de la vida y la subjetividad social al proceso productivo; el paso de las tecnologías disciplinarias de control a las tecnologías de seguridad; y, para el caso de los procesos de subjetificación laboral en la empresa flexible, el discurso del new management, que ha jugado un rol central en la institución de nuevas representaciones de lo que un sujeto debería aspirar a ser en tanto trabajador, emergiendo así un nuevo telos o ideal de trabajador emprendedor y flexible (Stecher, 2015).

De igual manera, las formas de gubernamentalidad que imperan en algunas organizaciones amplían el llamado para que los sujetos conduzcan todas sus actividades 
bajo criterios similares de eficiencia, algo así como una micro administración de la vida (Hassoun, 2012). Las nuevas formas de control estarían constituyendo nuevas subjetividades, trabajadores capaces de moverse rápidamente entre actividades y tareas en las que pueden ocupar una variedad de posiciones subjetivas, especialmente dentro del self-managing y el trabajo multifuncional en grupos o equipos. En este contexto, se pueden encontrar soluciones individualistas para manejar el estrés, sea resistiendo la presión de las circunstancias externas a través de esfuerzos internos o saliéndose de la presión externa invocando el compañerismo (Thunman, 2015).

En el marco de nuevas formas de trabajo, Dlaske (2016) encuentra que el workawayer es interpelado por una especie de humanismo cosmopolita no capitalista, con énfasis en los viajes como medio para lograr la comprensión intercultural y contribuir a una causa que vale la pena, entra gradualmente en sintonía y es entrenado en una variedad de prácticas y racionalidades que gobiernan, cada vez más, el mundo neoliberalizado contemporáneo del trabajo y la sociedad en su conjunto. El trabajador, un aventurero cultural, adquirirá así las habilidades necesarias para aplicaciones de trabajo eficaces y para la organización de viajes. En cuanto a las competencias y actitudes, los trabajadores se aproximan al yo empresarial, mostrando además flexibilidad, sensibilidad al riesgo y orientación al cliente, responsabilidad personal, orientación al objetivo y disposición a la acción (Dlaske, 2016).

En el contexto de la externalización de tareas, Ettlinger (2016) sugiere que la aceptación de un trabajo externalizado, ya sea por necesidad o por entretenimiento, no implica necesariamente satisfacción; este tipo de trabajo a menudo requiere una inversión personal y psicológica. En el marco de la transformación de empleados públicos a individuos business-like guiados por una lógica económica, y motivados por el servicio al cliente, a los sujetos se les estaría exigiendo equiparse con nuevas habilidades si quieren permanecer en su trabajo. Nyamori (2009) plantea que la primacía de lo económico sobre otras consideraciones, si bien podría contribuir al mejoramiento de los servicios, podría también ser una amenaza potencial al rol de las autoridades locales para posibilitar la democracia.

En el marco de algunos de los programas enfocados a promover el trabajo en los jóvenes, Assusa y Brandán (2014) plantean que el diagnóstico proporcionado por instancias políticas y científicas produce, aunque no de manera unilineal, las condiciones de una subjetividad laboral de su propia población objetivo, es decir, produce a este grupo como jóvenes inempleables. Así, uno de los aspectos más importantes de la formulación de políticas públicas es la forma en que las políticas construyen nuevas categorías de subjetividad, o bien, reconstituyen configuraciones subjetivas (Assusa y Brandán, 2014).Los discursos dirigidos a los jobseekers forman a los sujetos desempleados y los orientan como actores hacia el mercado laboral. Estos discursos 
proponen que el sujeto se venda como un producto para poder conseguir trabajo. De igual manera, están enfocados a inculcar el autocontrol, lo cual implica que las consecuencias psicológicas negativas del desempleo tienden a ser minimizadas, y a alentar a los sujetos a la búsqueda y a la competencia por cualquier trabajo, sin importar que tan precario sea. Los discursos pretenden que el sujeto posea la ética protestante del trabajo duro y confiable y la nueva ética del trabajo flexible y en equipo (Boland, 2016).

En los espacios educativos, específicamente en las universidades, Bill (2017) plantea que a medida que la universidad se construye como una entidad de negocios, se basa cada vez más en métricas para medir su valor. El aumento de la potencia informática ha permitido encontrar nuevas medidas de rendimiento que capturan y cuantifican los impactos de la investigación en la sociedad. Estas operaciones colapsan la distinción entre lo cualitativo y lo cuantitativo, y posiblemente obligan a nuevos tipos de desempeño académico que traen aparejadas nuevas subjetividades. De esta manera, el proceso de valorización y crítica de los juicios sociales sobre los productos y los resultados de la investigación se relaciona con nuevas categorías de identificación subjetiva (Bill, 2017). Los académicos se sienten cada vez más presionados por el contexto regulatorio y, por lo tanto, están descontentos con las políticas de evaluación y las regulaciones que dan forma a sus prácticas (Amigot y Martínez, 2013; Raaper, 2016). Raaper (2016) encuentra que, en su discurso, los académicos hacen uso de metáforas como una forma de expresar su confusión y frustración con el contexto político, como una forma de distanciarse del contexto regulatorio. El nuevo ideal de la subjetividad académica tiende a favorecer una mayor eficiencia, transparencia y escrutinio, mientras que los propios académicos se sienten cada vez más presionados y en oposición.

En el contexto de la educación básica, Alves y Barreto (2009) plantean que las políticas educativas para la educación básica en Brasil, desde la década de 1990, vienen introduciendo formas de control e intensificación del trabajo docente que toman como objeto de gobierno la subjetividad de las profesoras y las emociones en la enseñanza. Dichas políticas estimulan una moral de autorresponsabilización y culpa por parte de las profesoras, que, aliada al deterioro de los salarios y de las condiciones de trabajo, viene contribuyendo a la intensificación y autointensificación del trabajo docente y a la generación de frustraciones y desencantos.

Considerando la relación que existe entre poder y resistencia, en los casos estudiados por los investigadores se encuentran algunos ejemplos de prácticas o posibilidades de resistencia. Por su parte, Ball y Olmedo (2013) encuentran prácticas específicas de resistencia de un grupo de maestros que confrontan directamente el discurso y la promulgación del neoliberalismo y sus tecnologías. Boland (2016) 
encuentra que el enfoque de la gubernamentalidad centrado en el análisis de los consejos para los jobseekers tiene límites, lo más obvio es que los individuos pueden, de varias maneras, negociar y resistirse a ser subjetivados como tal.

Con respecto a posibilidades de resistencia, Ettlinger (2016) señala que la Web 1.o estuvo basada en la cognición, mientras que la 2.o estuvo basada en las redes, las cuales son, sin embargo, competitivas e individualistas. Por el contrario, la Web 3.0 del futuro podría estar basada en la cooperación entre individuos. De esta manera, quienes trabajan mediante la externalización abierta de tareas podrían dirigir su productividad hacia una nueva frontera de la resistencia digitalizada (Ettlinger, 2016).

En lugar de simplemente respaldar el tipo de subjetividad empresarial implicada en las políticas predominantes, Dey y Steyaert (2016) encuentran que los emprendedores sociales, en su mayoría, son reacios a identificarse con la invocación del poder gubernamental, a menudo rechazando la subjetividad ofrecida por el discurso. La ética, más que una propiedad del individuo, es una práctica a través de la cual los emprendedores sociales se involucran de una manera crítica y creativa con las relaciones de poder existentes, y producen activamente condiciones de libertad, tanto para ellos como para otros; esto sin suponer un espacio utópico de libertad más allá del poder. De igual forma, Dey y Steyaert (2016) encuentran que las prácticas de libertad pueden tomar tres formas. La primera es la práctica de la problematización, los emprendedores promulgan la libertad a través de relatos que socavan las concepciones dominantes de la realidad empresarial y la subjetividad. La segunda da cuenta de las prácticas de relación a través de las cuales los emprendedores forjan vínculos con otros que trascienden modelos jerárquicos e instrumentales de coexistencia. Y la tercera hace referencia a las prácticas de la afirmación reflexiva, los emprendedores eligen libremente identificarse con la subjetividad prescrita (Dey y Steyaert, 2016).

\section{Subjetividades determinadas o subjetividades emergentes: algunas ideas para la discusión}

En la literatura revisada hay una tendencia a plantear que formas particulares de gubernamentalidad forman/producen ciertas subjetividades; solo algunas investigaciones (Gómez et al., 2015; Stecher, 2015) plantean que formas de gubernamentalidad promueven/ofrecen ciertas subjetividades. Considerar que formas de gubernamentalidad promueven u ofrecen ciertas subjetividades constituye un paso para empezar a romper con las lógicas deterministas y de causalidad lineal para el abordaje de la subjetividad. En esta vía se podría plantear que formas de gubernamentalidad promueven u ofrecen condiciones de subjetivación a partir de las cuales emergerían (Deacon, 2013) ciertas subjetividades. Es así como la subjetividad daría cuenta de 
formas particulares de pensar, sentir y actuar respecto a uno mismo, los otros y el mundo (Stecher, 2013; 2015) que emergen de la colectividad, por tanto, la subjetividad es una forma de singularidad colectiva (Molina, 2015; 2016a; 2016b; 2016c).

Continuando con la discusión en torno a determinación y emergencia, se puede señalar que existe una relación entre el Estado, la sociedad civil y la economía: dependiendo de la forma como se relacionen, se puede hablar de neoliberalismo o de gobierno social. El neoliberalismo implica una relación economía, Estado y sociedad civil, en la que la primera ocupa un lugar preponderante; en contraste con el gobierno social, en el que el Estado es el que ocupa este lugar y por tanto tiene a su cargo diferentes funciones, entre estas la creación de oportunidades de trabajo. Se podría decir que el emprendimiento es una forma de gubernamentalidad neoliberal en la medida en que el Estado desplaza hacia el sujeto responsabilidades que otrora estaban a su cargo, por ejemplo, lo que tiene que ver con la generación de oportunidades de trabajo y las protecciones sociales que del trabajo se derivan (Castel, 1997; 2004). Desde una clave neoliberal, es el sujeto el que debe gestionar sus propias oportunidades de trabajo.

Ahora bien, no necesariamente la forma como se relacionan el Estado, la economía y la sociedad civil va a formar o producir -a modo determinista- unas subjetividades en particular. Lo que configura esta relación son condiciones de subjetivación a partir de las cuales podrían emerger diferentes subjetividades. A nivel de la sociedad civil, las comunidades, los grupos y los sujetos pueden estar gestando y emergiendo otras dinámicas distintas a las promovidas u ofrecidas. Por ejemplo, desde lo propuesto por las lógicas de la economía de mercado, se va a encontrar que los sujetos son tratados como medios para un fin, y no como fines en sí mismos (Etzioni, 2001). Pero ¿qué se puede encontrar a nivel de las comunidades y los grupos?, ¿se podría encontrar algo distinto? De igual manera, es importante tener presente que las condiciones de subjetivación propuestas por diferentes formas de gubernamentalidad se van a arraigar de manera distinta dependiendo de las particularidades de los contextos. Entonces, cabe mencionar que la nueva concepción de sujeto que trae consigo el neoliberalismo implica que el sujeto se haga cargo de sí mismo, no obstante, considerando lo anteriormente planteado, se podría decir que no existiría una sola manera de hacerse cargo de sí, sino que podrían emerger diferentes formas de hacerse cargo de sí mismo.

Aquí es donde es importante considerar el asunto de la emergencia y de las situaciones y giros inesperados planteados desde los límites de la analítica de la gubernamentalidad. Desde la analítica de la gubernamentalidad, el gobierno consiste en aquella modalidad de acción que se dirige a la conducción de la conducta 
de los individuos con respecto a fines específicos (Foucault, 2007). No obstante, no se puede asumir de antemano que en todos los casos las racionalidades de gobierno logran imponerse; siempre cabe la posibilidad de que los receptores de estas acciones actúen de otra manera (Martínez, 2016). El mismo Foucault reconocía que el ejercicio de la racionalidad de gobierno supone unos límites.

En el caso de la gubernamentalidad, su efectividad en la dirección de la conducta de los individuos no es algo que pueda darse por sentado, uno de los límites en el ejercicio de la gubernamentalidad reside en el hecho de que esta normalmente no alcanza lo que se propone (Martínez, 2016). Lo propuesto desde la gubernamentalidad puede tomar otras formas, inesperadas e imprevistas, dependiendo de las creencias, los valores y los intereses de los actores a quienes está dirigida; de los contextos particulares en los que tiene lugar; de la compleja red de relaciones de actores; y de las diferentes traducciones llevadas a cabo en dicha red (Agudo, 2009; Martínez, 2016). De ahí, la importancia de llevar el análisis más allá de los textos oficiales donde pueden estar plasmadas formas de gubernamentalidad, para centrarse en la manera como funcionan en la práctica, donde sus efectos inesperados aparecen no como desviaciones de su diseño inicial. El que los gobernados se constituyan a sí mismos como gobernables no puede darse por sentado a partir de intencionalidades inferidas de textos y discursos, sino que constituye un problema de investigación en lugares y grupos donde tiene lugar la acción política y económica (Agudo, 2009). De esta manera, es pertinente reconocer la capacidad de agencia de los actores implicados en el mundo de las políticas (Mosse, 2012).

Esto nos lleva a considerar la noción de traducción, en la medida en que esta permitiría dirigir la atención sobre la diferencia entre la racionalidad de una política social y la realidad de sus condiciones de instrumentación (Agudo, 2009) y aplicación. El concepto de traducción se refiere a todos los desplazamientos a través de otros actores cuya mediación es indispensable para que cualquier acción ocurra. La traducción es, por definición, siempre, un malentendido, en la medida en que los intereses de los actores son necesariamente divergentes. Nada es por sí mismo, lo mismo o distinto de algo más. No hay equivalentes, solo traducciones (Latour, 1987; 1988).

Para el caso específico de la tesis doctoral en la que este artículo tiene lugar, esto nos exhorta a realizar un análisis de discurso de la política pública de emprendimiento en Colombia y, luego, a girar la mirada hacia lo que acontece en la práctica con respecto al emprendimiento en nuestro país, lo cual exigiría conocer tanto el discurso como la experiencia de algunos emprendedores. Este acercamiento a los sujetos permitiría conocer las diferentes subjetividades que estarían emergiendo. 


\section{Referencias bibliográficas}

AGUDO SANCHÍZ, A. (2009). Conocimiento, lenguaje, poder e intermediación. Perspectivas contemporáneas en la antropología de las políticas públicas. Estudios Sociológicos, (79), 63-110.

ALVES, M. y BARRETO, S. (2009). Reforma educacional, intensificação e autointensificação do trabalho docente. Educação y Sociedade, 30(106), 63-85. https://doi.org/10.1590/So10173302009000100004

AMIGOT, P. y MARTÍNEZ, L. (2013). Gubernamentalidad neoliberal, subjetividad y transformación de la universidad. La evaluación del profesorado como técnica de normalización. Athenea Digital, 13(1), 99-120. Recuperado de: http://dialnet.unirioja.es/servlet/articulo?codigo $=4165304 \mathrm{yinfo}=$ resumenyidioma $=\mathrm{SPA}$

ASSUSA, G. y BRANDÁN ZEHNDER, M. G. (2014). «Salvar a la generación perdida»: gubernamentalidad, empleabilidad y cultura del trabajo. El caso de un programa de empleo para jóvenes en Argentina. Revista de Sociologia E Política, 22(49), 157-174. https://doi.org/10.159o/ So104-44782014000100009

BAILEY, P. L. J. (2015). Consultants of conduct: New actors, new knowledges and new «resilient» subjectivities in the governing of the teacher. Journal of Educational Administration and History, 47(3), 232-250. https://doi.org/10.1080/o0220620.2015.1038696

BALL, S. (2016). Subjectivity as a site of struggle: refusing neoliberalism? British Journal of Sociology of Education, 37(8), 1.129-1.146. https://doi.org/10.1080/01425692.2015.1044072

BALL, S. y OLMEDO, A. (2013). Care of the self, resistance and subjectivity under neoliberal governmentalities. Critical Studies in Education, 54(1), 85-96. https://doi.org/10.1080/17508 487.2013 .740678

BARDIN, L. (2002). Análisis de Contenido. Madrid: Ediciones Akal.

BENGTSSON, A. (2011). European policy of career guidance: The interrelationship between career self-management and production of human capital in the knowledge economy. Policy Futures in Education, 9(5), 616-627. https://doi.org/10.2304/pfie.2011.9.5.616

BILL, A. (2017). Counter-conduct in creative university research: deliberations on freedom. Higher Education Research y Development, 36(2), 241-254. https://doi.org/10.1080/07294360.2016.12 08158

BOCCHETTI, A. y OLIVEIRA, B. (2012). UM professor (SEMPRE) a formar: O governo das subjetividades docentes em programas especiais de formação. Curriculo Sem Fronteiras, 12(2), 376-392.

BOLAND, T. (2016). Seeking a role: disciplining jobseekers as actors in the labour market. Work, Employment y Society, 3o(2), 334-351. https://doi.org/10.1177/0950017015594097 
CASTEL, R. (1997). Las metamorfosis de la cuestión social. Una crónica del salariado. Barcelona: Paidós.

CASTEL, R. (2004). La inseguridad social ¿Quées estar protegido? Buenos Aires: Ediciones Manantial.

CASTRO-GÓMEZ, S. (2015). Historia de la gubernamentalidad I. Razón de Estado, liberalismo y neoliberalismo en Michel Foucault. Bogotá: Siglo del Hombre Editores.

DAHLSTEDT, M. y HERTZBERG, F. (2012). Schooling entrepreneurs: Entrepreneurship, governmentality and education policy in Sweden at the turn of the millennium. Journal of Pedagogy / Pedagogický Casopis, 3(2), 242-262. https://doi.org/10.2478/v10159-012-0012-x

DEACON, T. (2013). Naturaleza incompleta. Cómo la mente emergió de la materia. Barcelona: Tusquets.

DEY, P. y STEYAERT, C. (2016). Rethinking the Space of Ethics in Social Entrepreneurship: Power, Subjectivity, and Practices of Freedom. Journal of Business Ethics, 133(4), 627-641. https://doi. org/10.1007/s10551-014-2450-y

DLASKE, K. (2016). Shaping subjects of globalisation: At the intersection of voluntourism and the new economy. Multilingua, 35(4), 415-44o. https://doi.org/10.1515/multi-2015-0oo2

DLASKE, K., BARAKOS, E., MOTOBAYASHI, K. y MCLAUGHLIN, M. (2016). Languaging the worker: Globalized governmentalities in/of language in peripheral spaces. Multilingua, 35 (4), 345-359. https://doi.org/10.1515/multi-2015-0oo9

ERARANTA, K. y MOISANDER, J. (2011). Psychological Regimes of Truth and Father Identity: Challenges for Work/Life Integration. Organization Studies, 32(4), 509-526. https://doi. org/10.1177/0170840611400293

ETTLINGER, N. (2016). The governance of crowdsourcing: Rationalities of the new exploitation. Environment and Planning, 48(11), 2162-218o. https://doi.org/10.1177/0308518X16656182

ETZIONI, A. (2001). La tercera vía hacia una buena sociedad. Madrid: Editorial Trotta.

FAŸ, E., INTRONA, L. y PUYOU, F. R. (2010). Living with numbers: Accounting for subjectivity $\mathrm{in} /$ with management accounting systems. Information and Organization, $20(1), 21-43$. https:// doi.org/10.1016/j.infoandorg.2009.10.001

FOUCAULT, M. (2007). Nacimiento de la biopolítica. México D. F.: Fondo de Cultura Económica.

GÓMEZ, L., JÓDAR, F. y BRAVO, M. (2015). Gubernamentalidad neoliberal y producción de conocimiento en la universidad: Genealogía de una configuración subjetiva. Universitas Psychologica, 14(5), 1.735-1.750. https://doi.org/10.11144/Javeriana.upsy14-5.gnpc

GUTIÉRREZ MONCLUS, P. y PUJOL TARRÈS, J. (2016). Occupational Therapy: Autonomy, Governmentality and Subjectification. Revista de Estudios Sociales, (57), 68-77. https://doi. org/10.7440/res57.2016.05 
HASSOUN, D. (2012). Costly attentions: Governing the media multitasker. Continuum, 26(4), 653-664. https://doi.org/10.1080/10304312.2012.698041

HAY, S., KAPITZKE, C. y CBSE, F. (2009). «Smart state» for a knowledge economy: reconstituting creativity through student subjectivity. British Journal of Sociology of Education, 30 (2), 151-164. https://doi.org/10.1080/01425690802700206

LAALO, H. y HEINONEN, J. (2016). Governing the entrepreneurial mindset: Business students' constructions of entrepreneurial subjectivity. European Educational Research Journal, 15(6), 696-713. https://doi.org/10.1177/1474904116662288

LATOUR, B. (1987). Science in Action. How to follow scientists and engineers through society. Cambridge, Massachusetts: Harvard University Press.

LATOUR, B. (1988). The Pasteurization of France. Cambridge, Massachusetts and London: Harvard University Press.

LOLICH, L. (2011).....and the market created the student to its image and likening. Neo-liberal governmentality and its effects on higher education in Ireland. Irish Educational Studies, 3o(2), 271-284. https://doi.org/10.1080/03323315.2011.569145

MARTÍNEZ-BASALLO, S. P. (2016). Más allá de la gubernamentalidad: políticas de colonización y desarrollo rural en el piedemonte caqueteño (1960-1980). Universitas Humanística, (82), 135162. https://doi.org/10.11144/Javeriana.uh82.gpcd

MATZA, T. (2012). «Good individualism»? Psychology, ethics, and neoliberalism in postsocialist Russia. American Ethnologist, 39(4), 804-818. https://doi.org/10.1111/j.1548-1425.2012.01396.x

MOLINA, N. (2015). La subjetividad, polífonía social en el sujeto. En Universidad Pontificia Bolivariana - Bucaramanga. Bucaramanga.

MOLINA, N. (agosto de 2016a). Avatares de la subjetividad. Subjetividades en las Ciencias Sociales. Trabajo presentado en II Congreso Internacional de Ciencias Sociales y Humanas: Educación y Diversidades, Manizales, Colombia.

MOLINA, N. (2016b). De rojo a blanco, huellas y transformaciones. Cali.

MOLINA, N. (2016c). Quince años no es nada. Posibilidades de la psicología en el postacuerdo. Cali.

MORRISSEY, J. (2015). Regimes of performance practices of the normalised self in the neoliberal university. British Journal of Sociology of Education, 36(4), 614-634. https://doi.org/http://www. tandfonline.com/action/showCitFormats?doi=10.1080/01425692.2013.838515

MOSSE, D. (2012). Una etnografía de las políticas de la ayuda en la práctica. En Pérez, B. (ed.), Antropología y desarrollo. Discurso, prácticas y actores (pp. 258-281). Madrid: Los libros de la Catarata, Instituto Universitario de Desarrollo y Cooperación, Universidad Complutense de Madrid. 
MOTOBAYASHI, K. (2016). Language teacher subjectivities in Japan's diaspora strategies: Teaching my language as someone's heritage language. Multilingua, 35(4), 441-468. https://doi. org/10.1515/multi-2015-0011

NIESCHE, R. (2010). Discipline through documentation: A form of governmentality for school principals. International Journal of Leadership in Education, 13(3), 249-263. https://doi. org/10.1080/13603121003692967

NYAMORI, R. (2009). Construction and effects of markets in a local authority in New Zealand. Accounting, Auditing and Accountability Journal, 22(7), 1.055-1.086. https://doi. org/10.1108/09513570910987376

PELLANDINI-SIMÁNYI, L., HAMMER, F.y VARGHA, Z. (2015). The Financialization of Everyday life or the Domestication of Finance? Cultural Studies, 29(5-6), 733-759. https://doi.org/10.10 8o/09502386.2015.1017142

RAAPER, R. (2016). Academic perceptions of higher education assessment processes in neoliberal academia. Critical Studies in Education, 57(2), 175-19o. https://doi.org/10.1080/17508487.201 5.1019901

REICH, A. (2008). Intersecting work and learning: Assembling advanced liberal regimes of governing workers in Australia. Studies in Continuing Education, 30(3), 199-213. https://doi. org/10.1080/01580370802439912

ROSE, N. (1996). Governing «advanced» liberal democracies. En: A. Barry, T. Osborne y N. Rose (eds.). Foucault and political reason. Liberalism, neo-liberalism and rationalities of government (pp. 37-64). London: University College London (UCL) Press.

STECHER, A. (2013). Un modelo crítico-interpretativo para el estudio de las identidades laborales. Contribuciones a la investigación psicosocial sobre trabajo y subjetividad en América Latina. Universitas Psychologica, 12(4), 1.311-1.324. https://doi.org/10.11144/Javeriana.UPSY12-4.mcie

STECHER, A. (2015). La empresa flexible como dispositivo de gobierno. Aportes de la Analítica de la Gubernamentalidad al estudio de las subjetividades laborales en América Latina. Universitas Psychologica, 14(5), 1.779-1.794.

SUSPITSYNA, T. (2010). Accountability in American education as a rhetoric and a technology of governmentality. Journal of Education Policy, 25(5), 567-586. https://doi. org/10.1080/02680930903548411

THUNMAN, E. (2015). Managing stress: A matter of proactivity or trust? A thematic study of female- and male-dominated wedish work settings. Qualitative Research in Organizations and Management, $10(2), 134-152$. 
VARMAN, R., SAHA, B. y SKÅLÉN, P. (2011). Market subjectivity and neoliberal governmentality in higher education. Journal of Marketing Management, 27(11-12), 1.163-1.185. https://doi. org/10.1080/0267257X.2011.609134

WONG, P. N. (2010). Frontier Governmentality: The art of governing the self through the eyes of a philippine governing elite. Asia-Pacific Social Science Review, 10(103-126).

ZUCKERWISE, G. M. (2012). Governmentality in Amsterdam's Red Light District. City, 16(1-2), 146-157. https://doi.org/10.1080/13604813.2012.662365 